\title{
Erratum to: Identification and characterization of microRNAs at different flowering developmental stages in moso bamboo (Phyllostachys edulis) by high-throughput sequencing
}

\author{
Jian Gao ${ }^{1} \cdot$ Wei Ge $^{1} \cdot$ Ying Zhang $^{1} \cdot$ Zhanchao Cheng $^{1} \cdot$ Long Li $^{1} \cdot$ Dan Hou $^{1}$ • \\ Chenglin $\mathrm{Hou}^{2}$
}

Published online: 5 October 2015

(C) Springer-Verlag Berlin Heidelberg 2015

\section{Erratum to: Mol Genet Genomics \\ DOI 10.1007/s00438-015-1069-8}

The original version of this article does not contain any sequence data accession numbers.

The accession number of the Moso bamboo Transcriptome/Gene expression BioProject is PRJNA288852.

The miRNA sequences were deposited in GenBank under the following nucleotide sequence accession numbers: SRX1209547, SRX1209513, SRX1209460, SRX1209405, SRX1209350.

The online version of the original article can be found under doi:10.1007/s00438-015-1069-8.

Jian Gao

gaojianicbr@163.com

1 Key Laboratory of Bamboo and Rattan Science and Technology of the State Forestry Administration, International Centre for Bamboo and Rattan, No. 8 Futong Dongdajie, Wangjing, Chaoyang District, 100102 Beijing, People's Republic of China

2 Capital Normal University School of Life Science, No. 105, The Third Ring Road, Haidian District, 100048 Beijing, People's Republic of China 